\title{
ON SURFACES OF LOW GENUS WHOSE TWISTOR LIFTS ARE HARMONIC SECTIONS
}

\author{
KAZUYUKI HASEGAWA
}

Communicated by Vasil V. Tsanov

\begin{abstract}
We determine surfaces of genus zero in four-dimensional hyperkähler manifolds whose twistor lifts are harmonic sections. As corollary, we prove that a surface of genus zero in four-dimensional Euclidean space is twistor holomorphic if its twistor lift is harmonic section.
\end{abstract}

\section{Introduction}

The twistor lifts play an important role and have been studied for oriented surfaces in oriented four-dimensional Riemannian manifolds by many researchers. For examples, in [2], it is proved that any oriented surface admits a conformal, superminimal immersion into the four-dimensional sphere. The twistor space is endowed with an almost complex structure, which is integrable when the base manifold is self-dual (see [1]). Surfaces with holomorphic twistor lifts are called twistor holomorphic surfaces (see [6]). The surfaces whose twistor lifts are harmonic sections have been studied in [8] and [9]. If the ambient spaces are self-dual Einstein, the twistor lifts of twistor holomorphic surfaces are harmonic sections. Note that recently surfaces whose twistor lifts are harmonic sections have been studied from the view point of the integrable systems in [3] and [11].

In this paper, we announce results for surfaces of genus zero in four-dimensional hyperkähler manifolds whose twistor lifts are harmonic sections. If certain conditions for the curvature tensors are satisfied and the twistor lift is a harmonic section, then the mean curvature vector field is a holomorphic section of the normal bundle with respect to the Koszul-Malgarange holomorphic structure (see [3], [8] and [9]). In general, holomorphic sections are not parallel. It is well-known that a compact constant mean curvature surface of genus zero immersed in $\mathbb{R}^{3}$ is totally umbilic, which is proved by Hopf in 1951. This theorem has been generalized in various settings, like higher codimension cases (see [4]). In these generalizations results, the mean curvature vector field is assumed to be parallel with respect 
to the normal connection. If the mean curvature vector field is parallel, then the surface is totally umbilic using our result (see for example [4]). Thus our main theorem is a generalization of the Hopf's theorem to surfaces with holomorphic mean curvature vector fields. Moreover, we can also show that a Lagrangian surface of genus zero in complex Euclidean plane with conformal Maslov form is the Whitney sphere (see [5]).

\section{Harmonic Sections}

Throughout this paper, all manifolds and maps are assumed to be smooth. Let $E$ be a vector bundle over a manifold $M$ and $E_{x}$ the fiber of $E$ over $x \in M$. We write $T P$ for the tangent bundle of a manifold $P$. For vector bundles $E, E^{\prime}$ over $M$, we denote the homomorphism bundle whose fiber is the space of linear mappings $E_{x}$ to $E_{x}^{\prime}$ by $\operatorname{Hom}\left(E, E^{\prime}\right)$, and set $\operatorname{End}(E):=\operatorname{Hom}(E, E)$. The space of all sections of a vector bundle $E$ is denoted by $\Gamma(E)$. Let $\varphi: N \rightarrow M$ be a smooth map and $E$ a vector bundle over $M$. The pull back bundle of $E$ by $\varphi$ is denoted by $\varphi^{\#} E$.

In this section, we summarize the fundamental formulae for sections of the sphere bundles. Let $E$ be a Riemannian vector bundle with a fiber metric $g^{E}$ and a metric connection $\nabla^{E}$ over an $n$-dimensional Riemannian manifold $(M, g)$. Let $K^{E}: T E \rightarrow E$ be the connection map with respect to $\nabla^{E}$. The canonical metric $G$ on $E$ is defined by

$$
G(\zeta, \zeta)=g\left(p_{*}(\zeta), p_{*}(\zeta)\right)+g^{E}\left(K^{E}(\zeta), K^{E}(\zeta)\right)
$$

for $\zeta \in T E$, where $p: E \rightarrow M$ is the bundle projection. We note that the projection $p:(E, G) \rightarrow(M, g)$ is a Riemannian submersion with totally geodesic fibers. We call $\operatorname{ker} p_{*}\left(\right.$ respectively $\left.\operatorname{ker} K^{E}\right)$ the vertical (respectively horizontal) subbundle of $T E$. Set $U E(=U(E)):=\left\{u \in E ; g^{E}(u, u)=1\right\}$. The set of all sections $\xi \in \Gamma(E)$ such that $\xi(M) \subset U E$ is denoted by $\Gamma(U E)$. We define $H^{\nabla^{E}}$ by

$$
H^{\nabla^{E}}(X, Y) \xi:=-\nabla_{X}^{E} \nabla_{Y}^{E} \xi+\nabla_{\nabla_{X} Y}^{E} \xi
$$

for $X, Y \in \Gamma(T M)$ and $\xi \in \Gamma(E)$. The rough Laplacian $\bar{\Delta}^{\nabla^{E}}$ of $\nabla^{E}$ is defined by

$$
\bar{\Delta}^{\nabla^{E}}(\xi)=\sum_{i=1}^{n} H^{\nabla^{E}}\left(e_{i}, e_{i}\right)(\xi)=-\sum_{i=1}^{n}\left(\nabla_{e_{i}}^{E} \nabla_{e_{i}}^{E} \xi-\nabla_{\nabla_{e_{i}} e_{i}}^{E} \xi\right)
$$

for $\xi \in \Gamma(E)$, where $e_{1}, \ldots, e_{n}$ is an orthonormal frame of $(M, g)$. We assume that $M$ is compact. Let $e$ be the energy functional defined on the space of all 
smooth maps from $M$ to $U E$. For a section $\xi \in \Gamma(U E)$, the energy $e(\xi)$ is given by

$$
e(\xi)=\frac{n}{2} \operatorname{vol}((M, g))+\frac{1}{2} \int_{M}\left\|\nabla^{E} \xi\right\|^{2} \mathrm{~d} v
$$

where $\mathrm{d} v$ denotes the Riemannian measure of $(M, g)$ and $\operatorname{vol}((M, g))$ is the volume of $(M, g)$. We say that $\xi \in \Gamma(U E)$ is a harmonic section if $\xi$ is a stationary point of $\left.e\right|_{\Gamma(U E)}$. The second term of the right hand side in (1) is called the vertical energy of $\xi \in \Gamma(U E)$. In our restricted variational problem, the vertical energy is important. Obviously, if a section is a harmonic map in the usual sense, then it is a harmonic section. A section $\xi \in \Gamma(U E)$ is a harmonic section if and only if the equation

$$
\bar{\Delta}^{E}(\xi)=\left\|\nabla^{E} \xi\right\|^{2} \xi
$$

holds (see [15]).

\section{Twistor Spaces and Twistor Lifts for Surfaces}

Let $(\tilde{M}, \tilde{g})$ be an oriented four-dimensional Riemannian manifold. The Hodge star operator is denoted by $*$. Since $*^{2}=$ id for all two-forms, the bundle $\Lambda^{2}(\tilde{M})$ of all two-forms on $\tilde{M}$ is decomposed into

$$
\Lambda^{2}(\tilde{M})=\Lambda_{+}^{2}(\tilde{M}) \oplus \Lambda_{-}^{2}(\tilde{M})
$$

where $\Lambda_{ \pm}^{2}(\tilde{M})=\left\{\omega \in \Lambda^{2}(\tilde{M}) ; * \omega= \pm \omega\right\}$. Using the metrics, we can identify $\Lambda_{-}^{2}(\tilde{M})$ with a vector subbundle $Q$ of $\operatorname{End}(T \tilde{M})$. A section $J \in \Gamma(U Q)$ satisfies $J^{2}=-I, \tilde{g}(J X, J Y)=\tilde{g}(X, Y)$ for all $X, Y \in T \tilde{M}$ and $-\Omega_{J} \wedge \Omega_{J}=\mathrm{d} \mu$, where $\Omega_{J}$ is the fundamental form of $J$ and $\mathrm{d} \mu$ is the volume form of $\tilde{M}$ compatible with the orientation. Note that $Q$ is a parallel subbundle in $\operatorname{End}(T \tilde{M})$ with respect to the connection which is induced by the Levi-Civita connection $\tilde{\nabla}$ of $\tilde{M}$. We use the same letter $\tilde{\nabla}$ for the induced connection. The twistor space $Z$ over $\tilde{M}$ is the unit sphere bundle $U Q$ of $Q$. The bundle projection $p: Z \rightarrow \tilde{M}$ and the connection $\tilde{\nabla}$ induce the decomposition

$$
T Z=T^{h} Z \oplus T^{v} Z
$$

into the horizontal subbundle $T^{h} Z$ and the vertical subbundle $T^{v} Z$. On $Z$, a natural almost complex structure $J^{Z}$ is defined by $J^{Z}(X)=\left(J\left(p_{*}(X)\right)\right)_{J}^{h}$ for all horizontal vectors $X$ at $J \in Z$ and $J^{Z}(V)=J^{v}(V)$ for all vertical vectors $V$, 
where $(\cdot)^{h}$ stands for the horizontal lift and $J^{v}$ is the canonical complex structure on each fiber ( $\simeq$ the two-dimensional unit sphere). Let $\tilde{R}$ be the curvature tensor of $\tilde{g}$. The curvature tensor $\tilde{R}$ induces the endomorphism $\hat{R}$ of $\Lambda^{2}(\tilde{M})$. It is wellknown that $\hat{R}$ can be decomposed into

$$
\hat{R}=W^{+}+W^{-}+N+\frac{\tau}{6} \mathrm{id}
$$

where $W^{+}$(respectively $W^{-}$) is the self-dual (respectively anti-self-dual) part of the Weyl curvature tensor, $N$ represents the traceless Ricci part and $\tau$ is the scalar curvature. If $W^{-}=0$, then $\tilde{M}$ is called a self-dual manifold. The almost complex structure $J^{Z}$ on the twistor space is integrable if and only if $W^{-}=0$ (see [1]). If the Ricci tensor is proportional to the Riemannian metric, then $\tilde{M}$ is called an Einstein manifold.

Let $f:(M, g) \rightarrow(\tilde{M}, \tilde{g})$ be an isometric immersion from an oriented surface $(M, g)$ into an oriented four-dimensional Riemannian manifold $(\tilde{M}, \tilde{g})$. The Levi-Civita connection of $g$ is denoted by $\nabla$. Let $T^{\perp} M$ be the normal bundle of $f$ and $\nabla^{\perp}$ the normal connection of $T^{\perp} M$. We say that an orthonormal frame $e_{1}, e_{2}, e_{3}, e_{4}$ of $f^{\#}(T \tilde{M})$ is adapted if

1) $e_{1}, e_{2}, e_{3}, e_{4}$ is compatible with the orientation of $\tilde{M}$

2) $e_{1}, e_{2}$ is compatible with the orientation of $M$

3) $e_{3}, e_{4}$ are normal to $M$.

Using an adapted frame, we define $J: T M \rightarrow T M$ by $J\left(e_{1}\right)=e_{2}$ and $J\left(e_{2}\right)=$ $-e_{1}$, and $J^{\perp}: T^{\perp} M \rightarrow T^{\perp} M$ by $J^{\perp}\left(e_{3}\right)=-e_{4}$ and $J^{\perp}\left(e_{4}\right)=e_{3}$. It is evident that $\nabla J=0$ and $\nabla^{\perp} J^{\perp}=0$. We set

$$
\tilde{J}(X):=J(X) \text { and } \tilde{J}(\zeta):=J^{\perp}(\zeta)
$$

for $X \in T M$ and $\zeta \in T^{\perp} M$. Then $\tilde{J}$ is a section of $U\left(f^{\#} Q\right)\left(=f^{\#}(Z)\right)$ and $\tilde{J}$ is called the twistor lift of $M$. Hereafter, we often omit the symbol " $f$ " for the induced objects of the immersion $f$ if there is no confusion for the sake of simplicity. For example, we use the same letter $\tilde{\nabla}$ for the pull back connection $f^{\#} \tilde{\nabla}$ by $f$. We say that $M$ is a superminimal surface if the twistor lift is a horizontal map, that is, $\tilde{\nabla} \tilde{J}=0$. If $\tilde{J}_{*} \circ J=J^{Z} \circ \tilde{J}_{*}$ (precisely, $\left(f_{\#} \circ \tilde{J}\right)_{*} \circ J=J^{Z} \circ\left(f_{\#} \circ \tilde{J}\right)_{*}$, where $f_{\#}: U\left(f^{\#} Q\right) \rightarrow U Q$ is the bundle map), then $M$ is called a twistor holomorphic surface. Let $\alpha$ (respectively $H$ ) be the second fundamental form (respectively the 
mean curvature vector field) of $M$. We define a $T^{\perp} M$-valued symmetric tensor $B$ by

$$
B(X, Y)=\alpha(X, J Y)-J^{\perp} \alpha(X, Y)+J^{\perp} \alpha(J X, J Y)+\alpha(J X, Y)
$$

for all $X, Y \in T M$. We see that $M$ is twistor holomorphic if and only if $B=0$ (see [8]). Note that a surface is superminimal if and only if it is minimal and twistor holomorphic (see [6]).

\section{Surfaces Whose Twistor Lifts are Harmonic Sections}

Let $f:(M, g) \rightarrow(\tilde{M}, \tilde{g})$ be an isometric immersion from an oriented surfaces $(M, g)$ into an oriented four-dimensional Riemannian manifold $(\tilde{M}, \tilde{g})$. For $\beta \in$ $\Gamma\left(\operatorname{Hom}\left(T M \otimes T M, T^{\perp} M\right)\right)$, we define $\nabla^{\prime} \beta$ by

$$
\left(\nabla_{X}^{\prime} \beta\right)(Y, Z)=\nabla_{X}^{\perp} \beta(Y, Z)-\beta\left(\nabla_{X} Y, Z\right)-\beta\left(Y, \nabla_{X} Z\right)
$$

for all $X, Y, Z \in \Gamma(T M)$. A $T^{\perp} M$-valued one-form $\delta \beta$ is defined by

$$
(\delta \beta)(X)=-\sum_{i=1}^{2}\left(\nabla_{u_{i}}^{\prime} \beta\right)\left(u_{i}, X\right)
$$

for all $X \in T M$, where $u_{1}, u_{2}$ is an orthonormal frame of $M$. In [8], we have proved that the twistor lift $\tilde{J}$ is a harmonic section if and only if the equation

$$
(\delta \alpha)(J X)=J^{\perp}(\delta \alpha)(X)
$$

holds for all $X \in T M$. Obviously, the twistor lift of a superminimal surface is a harmonic section. In [9], we have proved

Theorem 1. Let $M$ be an oriented surface in a self-dual Einstein manifold. Then the following statements are mutually equivalent:

1) The twistor lift $\tilde{J}$ is a harmonic section

2) The mean curvature vector $H$ satisfies $\nabla_{J}^{\perp} H=J^{\perp} \nabla_{X}^{\perp} H$ for all $X \in T M$

3) $\delta B=0$.

Typical examples of self-dual Einstein manifolds are four-dimensional sphere $\mathbb{S}^{4}$, the projective plane $\mathbb{C P}^{2}$ and a K3-surface with an Einstein-Kähler metric relative to the opposite orientation of the canonical one. Let $\tilde{M}$ be a four-dimensional 
hyperkähler manifold which hyperkähler structure is generated by $I_{1}, I_{2}$ and $I_{3}$. If an orientation of $\tilde{M}$ is given by

$$
-\sum_{i=1}^{3} \Omega_{I_{i}} \wedge \Omega_{I_{i}}
$$

then we have $I_{1}, I_{2}, I_{3} \in \Gamma(Z)$, where $\Omega_{I_{i}}$ is the two form defined by $\Omega_{I_{i}}(X, Y)=$ $\tilde{g}\left(I_{i} X, Y\right)$ for $X, Y \in T M(i=1,2,3)$. We note that $\tilde{M}$ is a self-dual Einstein (in fact, Ricci flat) manifold with respect to this orientation. The twistor space $Z$ of $\tilde{M}$ is $\tilde{M} \times \mathbb{S}^{2}(1)$. Let $\hat{p}: Z \rightarrow \mathbb{S}^{2}(1)$ be the projection. We define the normal curvature $K^{\perp}$ by $K^{\perp}=\tilde{g}\left(R_{e_{1}, e_{2}}^{\perp} e_{4}, e_{3}\right)$, where $e_{1}, e_{2}, e_{3}, e_{4}$ is an adapted frame and $R^{\perp}$ is the curvature of the normal connection. The Euler characteristic of $T^{\perp} M$ is denoted by $\chi\left(T^{\perp} M\right)$. We have the following theorem.

Theorem 2. Let $\tilde{M}$ be a four-dimensional hyperkähler manifold and $M$ an oriented, connected, compact surface in $\tilde{M}$. If the twistor lift of $M$ is a harmonic section and the genus of $M$ is zero, then we have

1) $M$ is a non-superminimal minimal surface when $\chi\left(T^{\perp} M\right) \geq 4$

2) $M$ is a superminimal surface when $\chi\left(T^{\perp} M\right)=2$

3) $M$ is a non-superminimal twistor holomorphic surface when $\chi\left(T^{\perp} M\right) \leq 0$.

Note that $\chi\left(T^{\perp} M\right)$ is an even integer if $\tilde{M}$ is a hyperkähler manifold. For example this is true (see [12] and [13]) for minimal surfaces in hyperkähler manifolds, in particular, for K3 surfaces with the hyperkähler metric. In the case where $\tilde{M}$ does not admit any compact minimal surfaces, for example $\tilde{M}=\mathbb{R}^{4}$, we can obtain the following corollary immediately.

Corollary 3. Let $M$ be an oriented, compact, connected surface of genus zero in $\mathbb{R}^{4}$. If the twistor lift of $M$ is a harmonic section, then $M$ is a non-superminimal twistor holomorphic surface.

In general, the holomorphic mean curvature vector field is not parallel. In the case where the mean curvature vector is parallel, using Corollary 3 , we have the following corollary (see e.g. [4]).

Corollary 4. Let $M$ be an oriented, connected, compact surface of genus zero in $\mathbb{R}^{4}$. If the mean curvature vector field is parallel with respect to the normal connection, then $M$ is totally umbilic. 
It is easy to obtain the Hopf's theorem for a constant mean curvature surface of genus zero in $\mathbb{R}^{3}$ using by Corollary 4 . In fact, by considering the totally geodesic immersion from $\mathbb{R}^{3}$ to $\mathbb{R}^{4}$, the constant mean curvature surface can be seen as immersed surface in $\mathbb{R}^{4}$ with parallel mean curvature vector field. Thus our main theorem is a generalization of the Hopf theorem.

To compare Corollaries 3 and 4, we recall the Grassmannian manifold and the Gauss map. Let $G_{o}^{4,2}$ be the Grassmannian manifold of all oriented two-planes in $\mathbb{R}^{4}$. As a manifold $G_{o}^{4,2}$ is isomorphic to $\mathbb{S}_{+}^{2}(1) \times \mathbb{S}_{-}^{2}(1)$, where $\mathbb{S}_{ \pm}^{2}(1)$ are the unit spheres in the three-dimensional vector spaces of all self-dual and anti-selfdual two-forms, respectively. Let $p_{ \pm}: G_{o}^{4,2}\left(\cong \mathbb{S}_{+}^{2}(1) \times \mathbb{S}_{-}^{2}(1)\right) \rightarrow \mathbb{S}_{ \pm}^{2}(1)$ be the projection onto each factor in the product. On the other hand, the twistor space $Z$ of $\mathbb{R}^{4}$ is $\mathbb{R}^{4} \times \mathbb{S}^{2}(1)$. The projection from $Z$ onto $\mathbb{S}^{2}(1)$ is denoted by $\hat{p}$. The map $f_{\#} \circ \tilde{J}$ can be identified with the map $M \ni x \mapsto \omega_{1} \wedge \omega_{2}-\omega_{3} \wedge \omega_{4}$, where $\omega_{1}, \ldots, \omega_{4}$ is the dual basis of an adapted basis $e_{1}, \ldots, e_{4}$ at each point $x \in M$. Then we have

$$
\hat{p} \circ \tilde{J}=p_{-} \circ \varphi
$$

where $\varphi: M \rightarrow G_{o}^{4,2}$ is the Gauss map of $M$ in $\mathbb{R}^{4}$. Therefore, if the twistor lift of $M$ in $\mathbb{R}^{4}$ is a harmonic section, then a part of the Gauss map is harmonic. On the other hand, surfaces in Euclidean space whose (e) Gauss map is harmonic have parallel mean curvature vector fields (see [14]).

Finally, we give an application to a Lagrangian surface in $\mathbb{C}^{2}\left(\cong \mathbb{R}^{4}\right)$. Let $(\tilde{M}, \tilde{g}, \phi)$ be a four-dimensional Kähler manifold with the complex structure $\phi$ and denote the Kähler form of $M$ by $\Omega$. If a surface $M$ in $\tilde{M}$ is Lagrangian, that is, $\phi(T M)=$ $T^{\perp} M$, then we define the Maslov form $\omega$ on $M$ by $\omega(X)=(1 / \pi) \Omega(X, \phi H)$ for all $X \in \Gamma(T M)$. For a Lagrangian surface $M$, the Maslov form on $M$ is said to be conformal if the tangent vector $\phi H$ is a conformal vector field (see [5]). Define an immersion $w: \mathbb{S}^{2}(1) \rightarrow \mathbb{C}^{2}$ by

$$
w(x, y, z)=\frac{1}{1+z^{2}}(x(1+\sqrt{-1} z), y(1+\sqrt{-1} z)) .
$$

The immersion $w$ is called the Whitney immersion or the Whitney sphere, which is a Lagrangian immersion. Since the Whitney immersion is twistor holomorphic, it is a Willmore surface, that is, it is a stationary point for the Willmore functional. More details on these subjects can be found in [7]. By using Corollary 3, we have the following fact which is proved in [5].

Corollary 5. Let $M$ be an oriented, connected, compact, Lagrangian surface of genus zero in $\mathbb{C}^{2}$. If the Maslov form on $M$ is conformal, then $M$ is congruent to the Whitney immersion. 


\section{Acknowledgements}

The author would like to thank to organizers for giving him the opportunity to present his results at this conference. This work is partially supported by the Grant-in-Aid for Young Scientists (B) \# 20740046, the Ministry of Education, Culture, Sports, Science and Technology, Japan.

\section{References}

[1] Atiyah M., Hitchin N. and Singer I., Self-duality in Four-dimensional Riemannian Geometry, Proc. R. Soc. London Ser. A 362 (1978) 425-461.

[2] Bryant R., Conformal and Minimal Immersions of Compact Surfaces into 4-sphere, J. Diff. Geom. 17 (1982) 455-473.

[3] Burstall F. and Khemar I., Twistors, 4-symmetric Spaces and Integrable Systems, Math Ann. 344 (2009) 451-461.

[4] Chen B.-Y., Geometry of Submanifolds, Marcel Dekker, New York, 1973.

[5] Castro I. and Urbano F., Lagrangian Surfaces in the Complex Euclidean Plane with Conformal Maslov Form, Tohoku Math. J. 45 (1993) 565-582.

[6] Friedrich T., On Surfaces in Four-spaces, Ann. Global Anal. Geom. 2 (1984) 275-287.

[7] Garay O., Extremals of the Generalized Euler-Bernoulli Energy and Applications, JGSP 12 (2008) 27-61.

[8] Hasegawa K., On Surfaces whose Twistor Lifts are Harmonic Sections, J. Geom. Phys. 57 (2007) 1549-1566.

[9] Hasegawa K., Stability of Twistor Lifts for Surfaces in Four-dimensional Manifolds as Harmonic Sections, J. Geom. Phys. 59 (2009) 1326-1338.

[10] Hasegawa K., Surfaces in Four-dimensional Hyperkähler Manifolds whose Twistor Lifts are Harmonic Sections, preprint.

[11] Khemar I., Geometric Interpretation of Second Elliptic Integrable System, Diff. Geom. Appl. 28 (2010) 40-64.

[12] Micallef M. and Wolfson J., The Second Variation of Area of Minimal Surfaces in Four-manifolds, Math. Ann. 295 (1993) 245-267.

[13] Micallef M. and Wolfson J., Area Minimizers in a K3 Surface and Holomorphicity, Geom. Funct. Anal. 16 (2006) 437-452.

[14] Ruh E. and Vilms J., The Tension Field of the Gauss Map, Trans. Amer. Math. Soc. 149 (1970) 569-573. 
[15] Wood C., The Energy of Hopf Vector Fields, Manuscripta Math. 101 (2000) 71-88.

Kazuyuki Hasegawa

Institute of Human and Social Sciences

Faculty of Teacher Education

Kanazawa University

Kakuma-machi, Kanazawa

920-1192 Ishikawa

JAPAN

E-mail address: kazuhase@staff.kanazawa-u.ac.jp 